\title{
Design of Multifunctional Vehicle Interior Environment Monitoring System Based on Wireless Communication
}

\author{
Yuqi Yan *, Jialin Yang, Zhongxu Qin \\ Wuhan University of Technology, China \\ E-mail:*1814696080@qq.com \\ www.whut.edu.cn
}

\begin{abstract}
The interior environment of the car affects the driver's mental state to a certain extent, so it is necessary to design a multi-functional interior environment monitoring system. In this paper, STM32F103 single chip microcomputer is used as the core controller, which integrates multiple sensors to collect various environmental information in the vehicle, and transmits the data to the monitoring platform through WiFi wireless communication. When the detected data exceeds the preset threshold, the monitoring system will generate an alarm to remind the user to carry out relevant operations. The system has the advantages of low cost, convenient use and high precision, and has certain commercial value and market prospect.
\end{abstract}

Keywords: car, sensors, STM32F103, WiFi

\section{Introduction}

At present, the car is an indispensable means of transportation in daily life. The internal environment of the car affects the physical condition of the driver and passengers to a certain extent. Therefore, it is necessary to design a multifunctional vehicle interior environment monitoring system. The multi-function internal environment monitoring system can collect the temperature, humidity, $\mathrm{CO}$ concentration, PM2.5 concentration and other internal environment information in the car in real time, and display them on the LCD screen. When the detected data exceeds the preset threshold, the monitoring system will generate an alarm to remind the user to carry out corresponding operation. The system can also transmit various data information to the monitoring platform through $\mathrm{WiFi}$ wireless communication, and users can view the historical data information on the monitoring platform. The vehicle interior environment monitoring system can effectively monitor and alarm the vehicle interior environment (C) The 2021 International Conference on Artificial Life and Robotics (ICAROB2021), January 21 to 24, 2021 information in real time. The system is convenient to use and has a certain market value.

\section{Main control chip and peripheral circuit}

The overall hardware structure of the system is composed of main control chip and peripheral modules, as shown in Fig.1. The multifunctional internal environment monitoring system selects STM32F103VET6 single-chip computer as the core controller of the hardware control system. The processor of STM32F103VET6 is ARM Cortex-M3 high performance kernel, with a maximum operating frequency of $72 \mathrm{Mhz}$, Flash memory of $512 \mathrm{~KB}$, SRAM of $64 \mathrm{~KB}$, and encapsulated in LQFP64 ${ }^{1}$. Its peripheral configuration is strong and supports many communication interfaces including SPI interface, I2C interface, USB interface, USART interface, ADC, timer and other peripherals ${ }^{2}$. With $80 \mathrm{IO}$ ports, it is easy to connect peripheral modules for function expansion. The powerful function ensures that the chip can fully meet the hardware functional requirements of the whole system. 


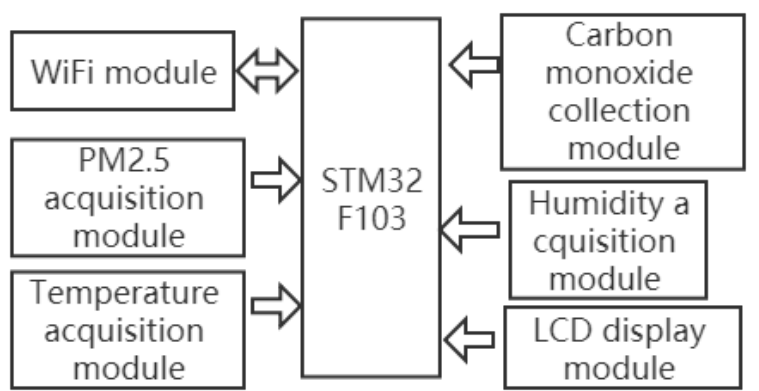

Fig.1 Overall architecture of hardware system

\subsection{AD converter circuit}

The STM32F103 series has three ADC, each of which has 16 conversion channels, with a conversion accuracy of 12 bits and a resolution of 1/4096. The ADC conversion circuit is shown in Fig.2. The dynamic contacts of the patch sliding rheostat of the AD conversion circuit in the monitoring and control system are connected to the ADC channel pins of the MCU chip. When the user rotates the sliding rheostat adjustment knob, the magnitude of the dynamic contact voltage in the $\mathrm{AD}$ conversion circuit part of the system will change accordingly. The range of voltage change is $0 \sim 3.3 \mathrm{~V}$, which is also the default ADC voltage collection range of the hardware end circuit board of the system.

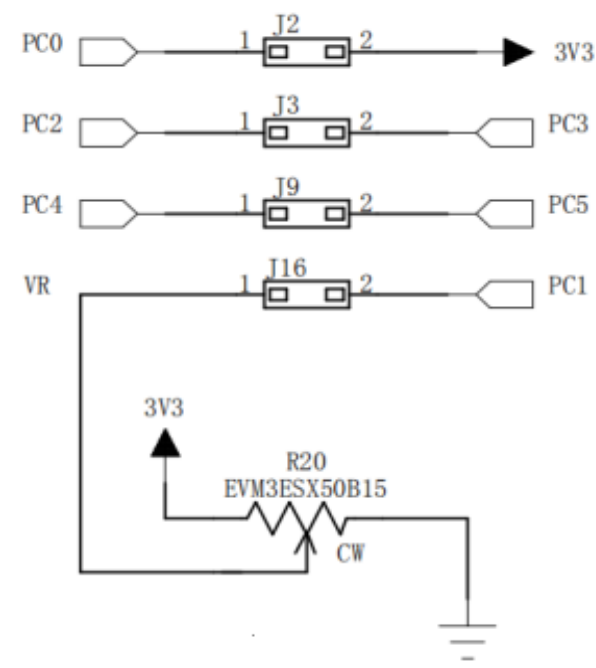

Fig.2. ADC converter circuit

\subsection{JTAG interface circuit design}

JTAG (Joint Test Action Group) is an international standard test protocol. It mainly tests the internal MCU chips of the monitoring and control system, and simulates and debugs the hardware control part of the system. As shown in Fig.3, there are four main lines in the JTAG interface part: TMS, TCK, TDI, TDO, which are mode selection, clock, data input and data output lines respectively. In the design of the monitoring and control system, the JTAG interface circuit uses 20-pin interface wiring connection. In order to enhance the anti-jamming capability of the download side of the system, ground wires are added between each signal line.

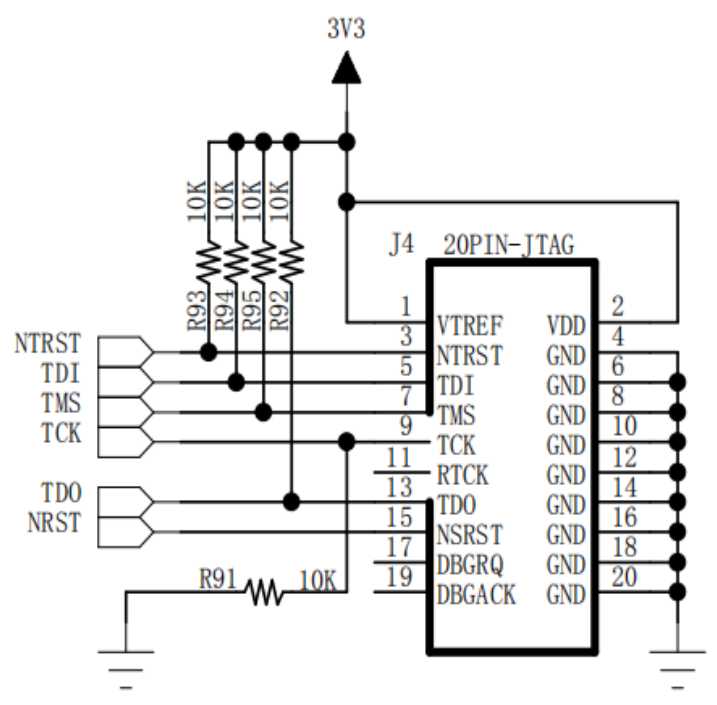

Fig.3. JTAG Download circuit

\section{Peripheral module of hardware system}

The peripheral modules of the hardware system mainly include temperature and humidity sensor, smoke sensor, interactive display screen, WiFi module and so on.

\subsection{Temperature and humidity sensor}

DHT11 digital temperature and humidity sensor is a temperature and humidity composite sensor with calibrated digital signal output. By using special digital module collection and temperature and humidity sensor technology, the environmental monitoring and control system has strong reliability and stability for temperature and humidity detection of the environment. The DHT11

(C) The 2021 International Conference on Artificial Life and Robotics (ICAROB2021), January 21 to 24, 2021 
module has low cost and strong anti-jamming capability, and it can measure the relative temperature and humidity of the automotive interior environment quickly. DHT11 uses full range calibration, which allows the module to be interchanged directly without having to be re-calibrated if a failure occurs. As shown in Fig.4, the DHT11 module contains a capacitive humidity sensor to measure ambient humidity and an NTC temperature sensor to measure ambient temperature. Besides, a small, high-performance 8 bit MCU is connected to the module.

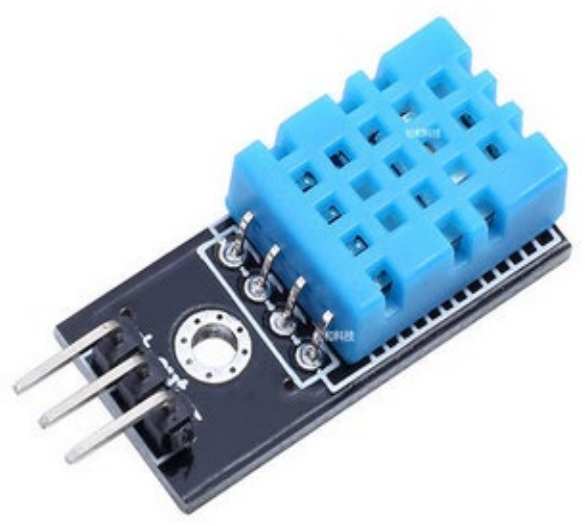

Fig.4. DHT11 sensor

\section{2. $M Q-2$ sensor}

The physical picture of the MQ-2 is shown in Fig.5. The MQ-2 gas sensor used in the environmental monitoring and control system can detect the presence of liquefied gas, butane, methane or alcohol in the environment which are potentially dangerous to human health. The conductivity of gas-sensitive materials increases with the increase of the concentration of combustible gas. The current smoke concentration value is output from the sensor signal by the conditioning circuit and sent to the MCU for processing. The alarm concentration of this sensor is 0 when it is not set, and the alarm concentration threshold can be set by the user with an upper limit of $100 \%$. The MQ-2 sensor has a wide detection radius, maintains high sensitivity and fast response in a variety of complex environments and excellent stability in harsh environments.

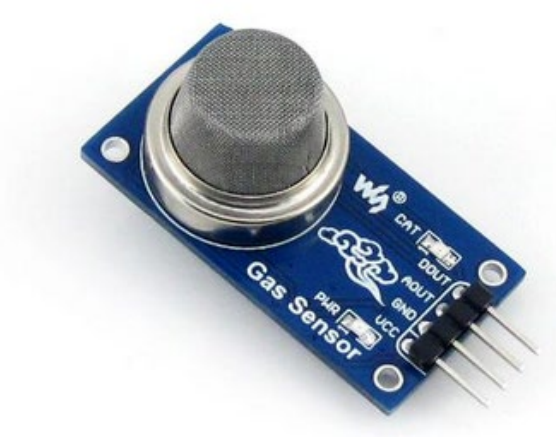

Fig.5. MQ-2 sensor

\subsection{ESP8266 module}

ESP8266 WIFI wireless communication module is selected as the data transmission bridge between smart hardware and server in this system, as shown in Fig.6. The ESP8266 module integrates a low power ESP8266 chip developed by LEXIN Information Technology Company. The chip is highly integrated with a built-in TCP/IP protocol stack and TR switches, amplifiers, regulators, power management components, etc. Its on-chip storage and processing power is strong, and it requires very few peripheral circuits, so it is easy to embed and develop ${ }^{3}$.

The ESP8266 module is powered by a $3.3 \mathrm{~V}$ voltage and operates in three modes: SoftAP mode, Station mode and SoftAP+ Station coexistence mode. In SoftAP mode, this module is equivalent to a wireless access point providing wireless access services, similar to a wireless router. Other terminal devices can connect to set up a LAN with ESP8266 for data transmission; in Station mode, this module is equivalent to a wireless terminal, which can connect to the Internet through a router and transmit data to the server; in SoftAP+Station coexistence mode, ESP8266 module can establish LAN communication with other terminal devices as a wireless access point, and can also connect services for Internet communication $^{4}$.

The ESP8266 module usually uses TCP protocol and UDP protocol for data transmission. UDP is a connectionless, unreliable protocol which does not require the receiver to confirm before sending the data and data transmission efficiency is high, but the reliability is low, which makes it unable to determine whether the data is complete in the transmission process. 
The TCP protocol is a link-oriented and reliable protocol which needs to wait for the receiver to confirm before the connection can be established. It is secure, reliable and the integrity of data can be guaranteed. The ESP8266 module in this system uses TCP protocol to set the WIFI application mode of ESP8266 as STA mode through AT instruction, and chooses TCP connection to realize the function of establishing data connection with the server.

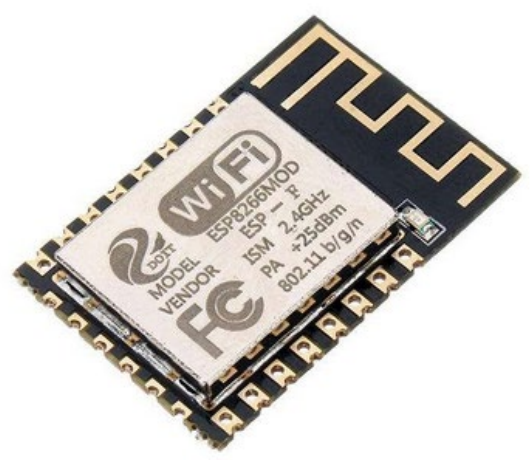

Fig.6. ESP8266 module

\subsection{User interactive display module}

The physical picture of the display is shown in Fig.7, which consists of an LCD display panel, a capacitive touch panel and a PCB substrate. The touch panel is equipped with a touch control chip, which is specially designed to handle the touch signals generated by the user when he touches the display, and then transmits the information through the outgoing signal lines. Because the MCU of the system cannot control the LCD panel directly, a LCD controller is needed to deal with the process of displaying. MCU only needs to give the data to the controller to display.

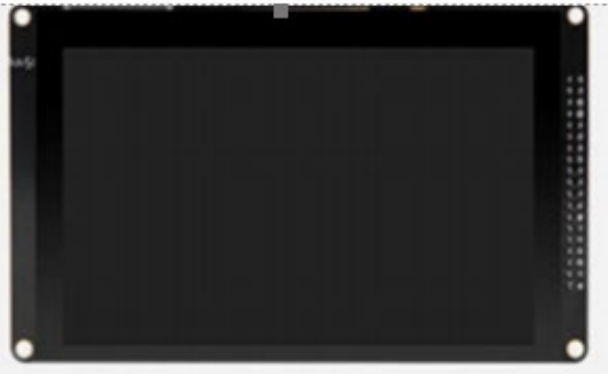

Fig.7. User interactive display module

\section{Software development environment}

This system chooses to develop the STM32F103VET6 controller in Keil5 development environment, and chooses $\mathrm{C}$ language as the development language. Keil5 is an integrated development environment with powerful compilation and simulation debugging capabilities.

STM32 is developed in two ways: register development and library development. This system is developed in the way of Library development, calling function interface directly to configure registers, which is fast and readable. Use STMISP downloader to program the STM32 core controller, and use TCP serial debugging assistant for functional testing.

The core controller of STM32 needs to collect data information of each sensor module, and control each peripheral module to realize the sub-functions of the system through internal logical analysis and processing.

\section{Conclusion}

The multi-functional vehicle interior environment monitoring system designed in this paper can achieve the functions designed in advance after testing. The LCD can display the data information collected by each sensor in real time. When the data exceeds the set threshold, the system will give an alarm.Through the serial debugging assistant, the WiFi communication function is normal, and the hardware device can connect and send data to the cloud server through WiFi module. The monitoring system designed in this paper has certain practical value.

\section{References}

1. Ibrahim, Dogan, Applying the ESP8266 processor in IoT application, Electronics World, 2017, 123(1976): pp.14-17.

2. Honghua Liao, Hao $\mathrm{Fu}$, Weichuang $\mathrm{Yu}$, et al., Design and Implement of Pyroelectric Energy Harvester Experimental Measurement System Based on STM32F103VET6, Sensors \& Transducers, 2016, 96(1): pp.69-74.

3. Fengzhi Dai, Yuxing Ouyang, Yiqiao Qin, et al., Development of Integral Smart Home Appliances, Journal of Robotics, Networking and Artificial Life, 2018, 4(4): pp.291-294.

4. Craig A, Lindley, Meet the ESP8266, Nuts \& volts, 2015, 36(10): pp.42-47. 